\title{
Cold and Hot Spots: From Inhibition to Enhancement by Nanoscale Phase Tuning of Optical Nanoantennas
}

\author{
Nicola Palombo Blascetta, Pietro Lombardi, Costanza Toninelli, and Niek F. van Hulst* \\ Cite This: Nano Lett. 2020, 20, 6756-6762 \\ Read Online
}

ABSTRACT: Optical nanoantennas are well-known for the confinement of light into nanoscale hot spots, suitable for emission enhancement and sensing applications. Here, we show how control of the antenna dimensions allows tuning the local optical phase, hence turning a hot spot into a cold spot. We manipulate the local intensity exploiting the interference between driving and scattered field. Using single molecules as local detectors, we experimentally show the creation of subwavelength pockets with full suppression

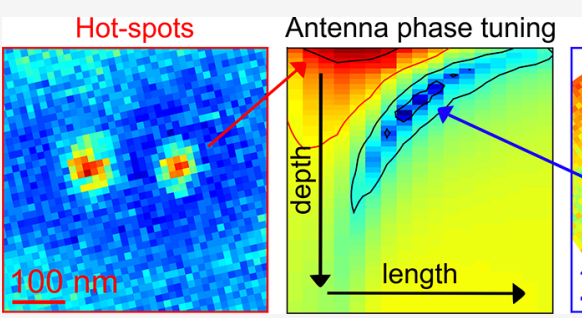
Cold-spots of the driving field. Remarkably, together with the cold excitation spots, we observe inhibition of emission by the phase-tuned nanoantenna. The fluorescence lifetime of a molecule scanned in such volumes becomes longer, showing slow down of spontaneous decay. In conclusion, the spatial phase of a nanoantenna is a powerful knob to tune between enhancement and inhibition in a 3-dimensional subwavelength volume.

KEYWORDS: local phase, near field interference, hot spot - cold spot, antenna enhancement, inhibition of emission, local density of states, superresolution, nanoantenna, plasmonics, single molecule

$\mathrm{I}^{\mathrm{t}}$

$t$ is well understood that the emission of molecules or atoms is directly influenced by their immediate surrounding, equivalent to the radiation resistance of an antenna depending on its environment. ${ }^{1-3}$ Indeed, in his 1966 milestone experiment, Karl-Heinz Drexhage placed $\mathrm{Eu}^{+}$atoms in front of a mirror and showed the luminescence lifetime becoming longer or shorter depending on the relative distance. This was intuitively explained as the $\mathrm{Eu}^{+}$atom interfering with "its own mirror image". ${ }^{2}$ More strictly, as pointed out by Purcell, ${ }^{1}$ the local vacuum electromagnetic field, which is coupled to the molecular transition dipole, is altered, thus inhibiting or accelerating the radiative transition. ${ }^{3}$ A large variety of optical cavity systems has been designed to control such coupling: concave mirror (laser) cavities, microresonators, ${ }^{4-6}$ photonic crystals with defects, ${ }^{7,8}$ and many more. Photonic crystal cavities, with typically wavelength dimensions (diffraction limited), have been proven valuable to reduce the local density of states and thus inhibit radiative decay..$^{9-11}$ Beyond the diffraction limit, metallic plasmonic designs are exploited, such as surface plasmon polariton cavities ${ }^{12,13}$ or true localized plasmon nanoantenna systems such as spheres, cubes, and rods. ${ }^{14-16}$ Some indications of inhibition were reported. ${ }^{17,18}$ Ultimate confinement of the electromagnetic field has been achieved in gap-plasmon systems, with Purcell enhancement up to even a million times. ${ }^{19}$

Most works on enhancing the emitters' radiative decay rate focus on minimizing the mode volume, tuning the resonance, and improving the resonator quality, achieving high Purcell factors in the range from $10^{2}$ to $10^{4} \cdot{ }^{20,21}$ Indeed, nanoantenna hot spots have been shown to produce record enhancement, intense SERS signal, and even single-molecule strong-coupling at room temperature. ${ }^{19}$ Besides the high local field, the local phase is equally important. For reflection at a mirror or interface the phase step is fixed, zero, or $\pi$, depending on transition to a low or high index medium. For a photonic crystal, the reflection phase can be changed from zero to $\pi$ by tuning the wavelength through the stop band. ${ }^{22-24}$ For plasmonic antennas, the local phase depends strongly on specific shape and resonance conditions, providing even larger flexibility to engineer the local field. Indeed, plasmonic phased arrays, and planar optical components based on arrays of antennas ("metasurfaces") have proven effective for spatial beam shaping and directional control. ${ }^{25,26}$ Surprisingly, the local phase has hardly been exploited to control the actual coupling to photon emitters.

Here we present phase tuning of local spots at a nanoantenna. Changing the antenna length, we tune the hot spot out of phase, creating a nanoscale pocket with full suppression of the excitation field: a cold spot. ${ }^{27}$ We map the subwavelength cold spots using single molecules as local field probes, and show the importance of balance between phase and amplitude to create such cold spots. Next, turning from

Received: June 26, 2020

Revised: August 14, 2020

Published: August 17, 2020 


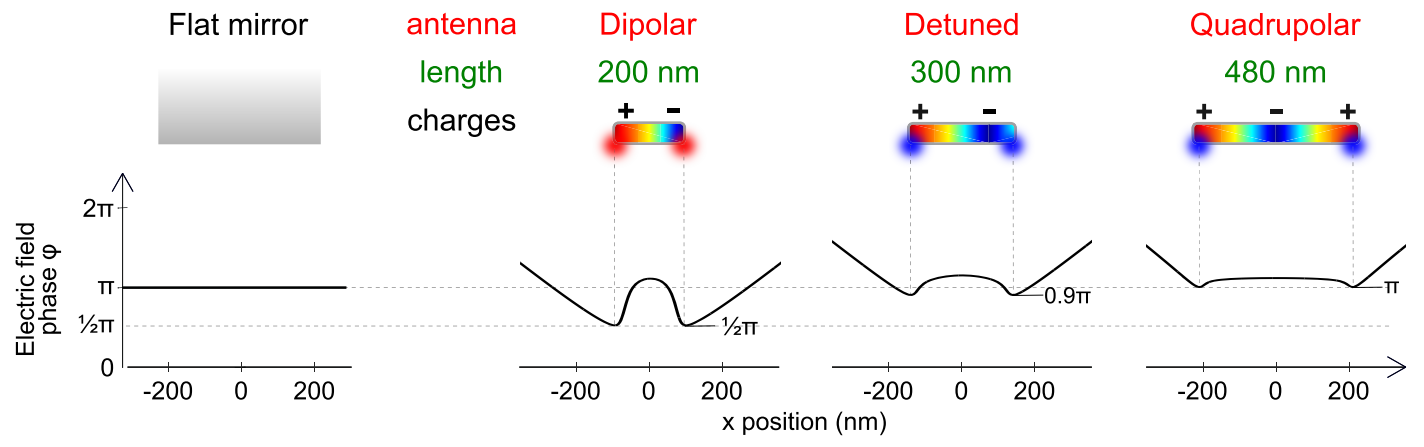

Figure 1. Phase of the optical field scattered at a nanoantenna. The phase shift $\phi$ of a plane-wave reflected on a flat metallic mirror, is simply a $\pi$ phase shift at the surface. The phase shift $\phi$ profiles for nanoantennas of increasing lengths, $200 \mathrm{~nm}$ (dipolar), $300 \mathrm{~nm}$ (detuned), and $480 \mathrm{~nm}$ (quadrupolar) are position dependent. The phase shift $\phi$ at the antenna field maxima changes continuously from $1 / 2 \pi$ to $\pi$ upon increasing the antenna length. On each antenna is sketched the typical charge oscillation, due to the resonance condition, together with the charge accumulation points. The incoming field is linearly polarized along the main antenna axis.

the excitation to the emission, we present the first inhibition of emission close to a plasmonic nanoantenna. Tuning the phase of the antenna, we create subwavelength volumes in which the fluorescence lifetime of a single molecule is lengthened, confirming the slowdown of radiative decay. Thus, we present Drexhage's experiment revisited, with a single molecule, in three dimensions and in a subwavelength volume.

Consider light reflected from a flat metallic mirror. An incident plane wave is reflected, maintaining a plane wavefront, acquiring a fixed phase shift $\phi=\pi$ (Figure 1). A metallic nanoantenna scatters the incident field with a phase $\phi$ and amplitude that are structured on a subwavelength scale, depending on the shape, size, and resonance wavelength of the nanoantenna. In Figure 1 we show the phase profile of the scattered field for $\mathrm{Al}$ rod antennas of 200, 300, and $480 \mathrm{~nm}$ length, for an incident plane wave $(\lambda=780 \mathrm{~nm})$ polarized along the rod. The $200 \mathrm{~nm}$ antenna supports a $\lambda / 2$ mode (dipolar resonance) and introduces a localized phase $\phi=1 / 2 \pi$ at the rod ends, the hotspots of the dipole antenna. The 300 $\mathrm{nm}$ antenna is detuned with respect to the incident field (outof-resonance) and the scattered field acquires a larger phase shift, $\phi=0.9 \pi$, at its ends. The scattered field becomes completely out-of-phase, $\phi=\pi$, for the $480 \mathrm{~nm}$ long rod, which supports a $\lambda$ mode (quadrupolar resonance). Moreover, the scattered phase also changes depending on the relative position to the antenna. The creation of such localized phase patterns is relevant for the tailoring of both the local excitation field and the local density of states (LDOS) experienced by a local emitter. The effective excitation field is the sum of the driving and the local scattering fields, which will interfere constructively or destructively depending on their relative amplitude and phase, generating subwavelength "hot spots" or "cold spots", respectively (depicted in Figure 1 as red and blue spots). Thus, the fact that amplitude and phase of the field scattered by antennas are both position- and resonancedependent provides a unique knob for interference and LDOS control on a subwavelength scale, through antenna detuning.

First, we analyze the effect of detuning on the constructive and destructive near-field interference. Specifically, we compare the local field around a resonant and a detuned antenna. In Figure 2 the electric field intensity distribution is plotted in the near-field of a $200 \mathrm{~nm}$ long dipole rod antenna and for a $300 \mathrm{~nm}$ detuned antenna. The $x$-component of the total electric field intensity $\left(I_{x}\right)$, as determined from FDTD simulations, is plotted, normalized to the incoming beam
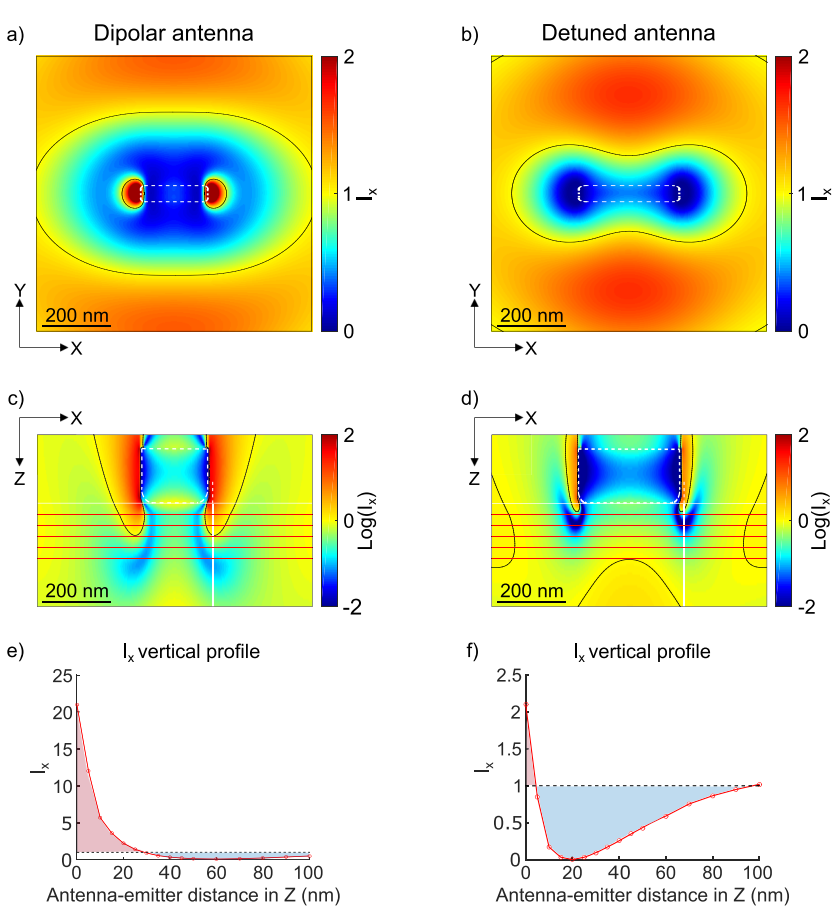

Figure 2. Electric-field intensity around a resonant dipole (left) and a detuned (right) nanoantennas. (a, b) Calculated maps of $x$-field intensity $15 \mathrm{~nm}$ under a $200 \mathrm{~nm}$ length (dipolar) and a $300 \mathrm{~nm}$ length (detuned) nanoantenna, respectively. (c, d) Calculated vertical $(x-z$ plane) maps of the $x$-field intensity for dipolar and detuned antenna, respectively. The antenna's position is indicated by dashed lines. The $\mathrm{Al}$ antennas $\left(50 \times 50 \mathrm{~nm}^{2}\right.$ cross-section $)$ are excited with a plane wave at a wavelength of $750 \mathrm{~nm}$, linearly polarized along the $x$-direction, the main antenna axis. The maps have been stretched in the $z$ direction ( $x: z$ aspect ratio 1:5) for the sake of clarity. Red horizontal lines are separated at $z$ distances of $10 \mathrm{~nm}$ from each other. The horizontal red lines are spaced $10 \mathrm{~nm}$ along $z$. (e, f) Calculated $x$-field intensity profiles in $z$, along the white vertical lines depicted in (c) and (d). $z=0$ is located at the metal interface of the antenna. The profiles show $z$-ranges with $I_{x}$ enhanced $(>1)$ and reduced $(<1)$, marked in red and blue, respectively. All calculations are performed with FDTD, for $750 \mathrm{~nm}$ wavelength, with linearly polarized light along the antenna axis. The field intensities are normalized to the incoming beam; the black contour lines correspond to scattering intensity equal to unity.

intensity $\left(I_{\text {inc }}\right)$. For the $200 \mathrm{~nm}$ dipolar antenna, one appreciates the two bright spots (hot spots with $I_{x}>I_{\text {inc }}$ ), with excitation enhancement $3.5 \times, 50 \mathrm{~nm}$ fwhm, and $220 \mathrm{~nm}$ 
separation. The antenna field is surrounded by a bright external ring at a distance of $\sim \lambda / 2$ in the $x-y$-plane. The solid black line marks the unity contour with $I_{x}=I_{\text {inc }}$. Remarkably, within the unity contour, a large central part of the intensity map shows areas with reduced excitation intensity, where the field is less intense than the incoming beam, $I_{x}<I_{\text {inc }}$ as result of interference with the incoming beam. For the detuned $300 \mathrm{~nm}$ antenna, the in-plane $I_{x}$ map looks radically different. The center of the map, instead of two bright "hot spots", now shows two dark spots, "cold spots", with local intensity $I_{x}=$ $10^{-5}$ far below the incident intensity $I_{\text {inc }}$ with $100 \mathrm{~nm}$ fwhm and $320 \mathrm{~nm}$ separation. The cold spots are slightly larger in size than the hot spots of the resonant case but nicely show that local enhancement can be turned into local reduction. The two cold spots are partially connected by a thinner area, also of reduced intensity. Compared to the dipolar resonant case, the unity contour is now shifted closer to the central spots, while also for detuned antenna we find a bright external ring at $\sim \lambda / 2$ from the center of the intensity map.

Figure $2 \mathrm{~b}$ shows vertical $x-z$ maps of the field intensity $I_{x}$. In very close proximity to the metal, the resonant dipole antenna generates over $100 \times$ excitation enhancement. The black unity contour line $\left(I_{x}=I_{\text {inc }}\right)$ nicely outlines the enhanced zones at antenna edges, extending until $z=30 \mathrm{~nm}$ below. The vertical map for the detuned antenna is markedly different. First of all, we notice how $I_{x}$ is much lower compared to the resonant case, with tiny unity contour lobes, much closer to the antenna edge and extending very shortly in the $z$-direction $(<5 \mathrm{~nm})$. Outside these areas, the detuned antenna mainly generates $I_{x}$ reduction $\left(I_{x}<1\right)$, with two very dark lobes separated $320 \mathrm{~nm}$ in $x$ and at a depth of $z=10-30 \mathrm{~nm}$. These lobes are the zones of full destructive interference between the incoming and scattered light and correspond to the two dark areas (cold spots) shown in the in-plane map.

For a quantitative comparison, in Figure $2 c$, we plot the vertical profiles of $I_{x}$ along the white vertical lines in Figure Figure $2 \mathrm{~b}$, at 110 and $160 \mathrm{~nm} x$-distance from the antenna center, for hot spot and cold spot, respectively. The dipolar antenna mainly provides strong $I_{x}$ enhancement for short-range interaction, until $30 \mathrm{~nm}$ where the incident field becomes suppressed. For the detuned antenna, the field is suppressed already at $10 \mathrm{~nm}$, with maximum suppression $\left(10^{-5}\right)$ predicted for $z=20 \mathrm{~nm}$, extending until very deep $\sim 100 \mathrm{~nm}$.

To investigate how the local antenna phase can turn local field enhancement into suppression, we consider the antenna rod length as a continuous variable. Figure 3 shows the vertical $(z$-direction, in green in Figure 3a) profile of both field intensity and phase while tuning the antenna length continuously $100-500 \mathrm{~nm}$. Panels b-d of Figure 3 show the scattered intensity $I_{\text {scat }}$ its phase $\phi$, and the total intensity $I_{x}$, respectively, all for the $x$-field component, with intensities on a log-scale. The intensity of the scattered light $I_{\text {scat }}$ (Figure $3 \mathrm{~b}$ ) shows a clear maximum for the dipole antenna length of 200 $\mathrm{nm}$, which decreases when tuning the length out of dipolar resonance. The unity intensity contour $\left(I_{\text {scat }}=I_{\text {inc }}\right)$ is indicate in red. The phase $\phi$ of the scattered $x$-field (Figure $3 \mathrm{c}$ ) shows a strong dependence on both depth $z$ and length. For $z=0$ the phase $\phi$ increases from $\phi=0$ for a very short antenna, to $\phi=$ $\pi / 2$ for the dipolar antenna, to a value of $\phi=\pi$ for a length of $480 \mathrm{~nm}$, the quadrupole antenna. At the same time, $\phi$ increases with $z$, due to the delay accumulated by propagation in space. As a result, the contour line $\phi=\pi$ extends through both depth and length (red line in Figure 3c). a)
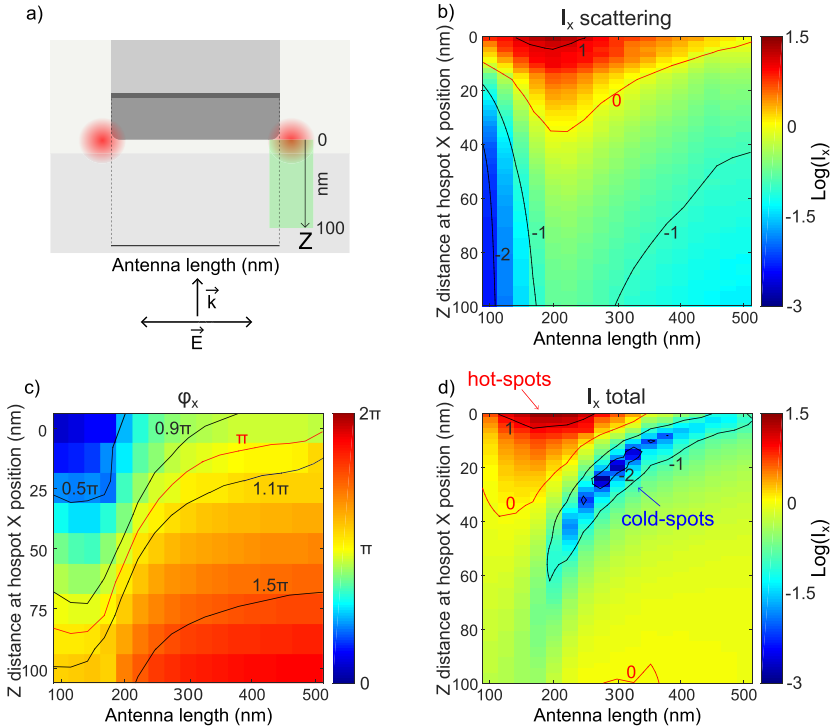

Figure 3. Phase tuning the near field interference, from hot to cold spots. (a) Sketch of a rod antenna located at $10 \mathrm{~nm} z$-distance from a glass substrate. The green area indicates the $z$-direction along which intensity and phase profiles are calculated. Calculated $x$-field scattered light intensity (b), scattered light phase with respect to the incident field (c), and total light intensity (d) for increasing antenna lengths and distance $z$. In (b) and (d) the red lines represent the contours where intensity is equal to incoming light. In (c) the red line represents the contour where scattered light phase is $\pi$. The total field (d) distinguishes the hot-spot area around the resonant dipole antenna with strong field enhancement and a more extended coldspot area around the detuned antenna with strongly reduced field intensity. FDTD calculations of the field $I_{x}$, and its phase $\phi$, performed at $750 \mathrm{~nm}$ wavelength, with linearly polarized light along the antenna axis. The $x$-field intensities are normalized to the incoming beam.

The total intensity $I_{x}$ (Figure 3d) shows the effective excitation field due to interference of $I_{\text {scat }}$ with $I_{\text {inc }}$. The phasedependent depth-length regions lead to hot and cold spots. The hot-spot region is centered at the $200 \mathrm{~nm}$ length dipolar antenna, with almost two-orders of magnitude enhancement at $z=0$. The cold-spot zone is wider, from 200 to $400 \mathrm{~nm}$ length detuned antenna and extends until $z=100 \mathrm{~nm}$. Most excitation reduction $\left(I_{x} \ll 1\right)$ occurs for a $300 \mathrm{~nm}$ antenna at $z$ $=25 \mathrm{~nm}$, with $I_{x}=10^{-5}$. The hot-spot region in Figure $3 \mathrm{~d}$ is mainly dictated by the strong intensity $I_{\text {scat }}$, with $I_{\text {scat }} \gg I_{\text {inc }}$ and negligible interference. On the other hand, the cold spots are located where we obtain full destructive interference between $I_{\text {scat }}$ and $I_{\text {inc }}$ hence at locations where $I_{\text {scat }}=I_{\text {inc }}$ and $\phi$ $=\pi$. Thus, the area with minimal $I_{x}$ in Figure $3 \mathrm{~d}$ corresponds to the region where the contour $I_{\text {scat }}=1$ in Figure $3 \mathrm{~b}$ crosses the contour $\phi=\pi$ in Figure 3c. By tuning the antenna phase right, one can obtain $100 \%$ full destructive interference. Moreover, the cold spot can be pulled into the near field zone, at only 25 $\mathrm{nm}$ distance, with a fwhm in depth of $20-30 \mathrm{~nm}$.

We now turn to the experiment. Aluminum nanoantennas of 200 and $300 \mathrm{~nm}$ in length are fabricated on a glass support by focused-ion-beam milling, to accurately define the proper length for phase control (Figure 4a,b). To probe the field around the antenna, we use a single fluorescent molecule: dibenzoterrylene (DBT) embedded in anthracene (AC) thin crystals $^{28}$ (see the Supporting Information). DBT molecules are all oriented parallel to each other along the flake, ${ }^{29}$ 
a)
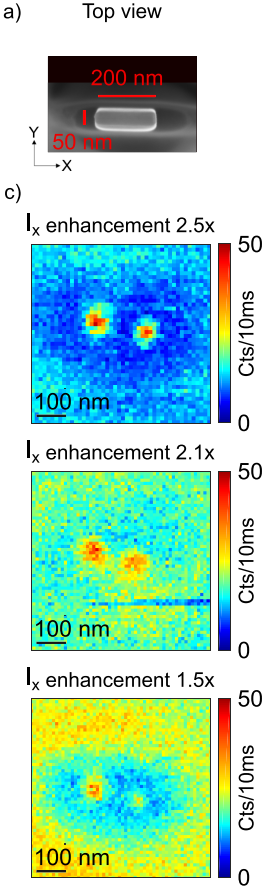

$I_{x}$ enhancement 1.1x
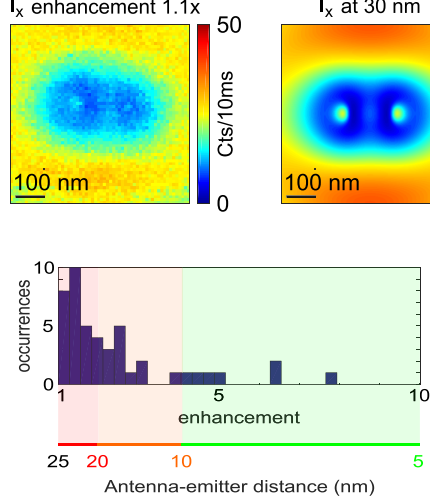
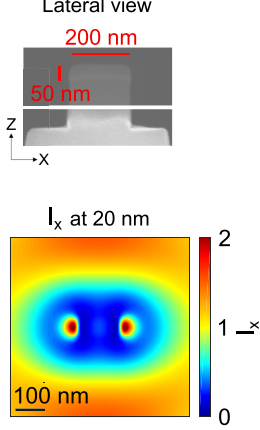

$\mathrm{I}_{\mathrm{x}}$ at $22 \mathrm{~nm}$

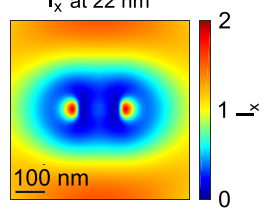

$\mathrm{I}_{\mathrm{x}}$ at $25 \mathrm{~nm}$

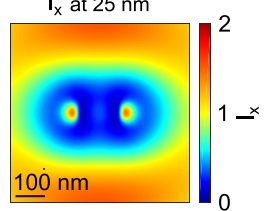

$\mathrm{I}_{\mathrm{x}}$ at $30 \mathrm{~nm}$

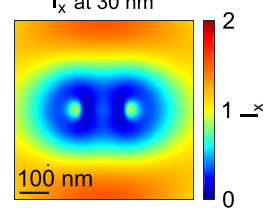

$100 \mathrm{~nm}$
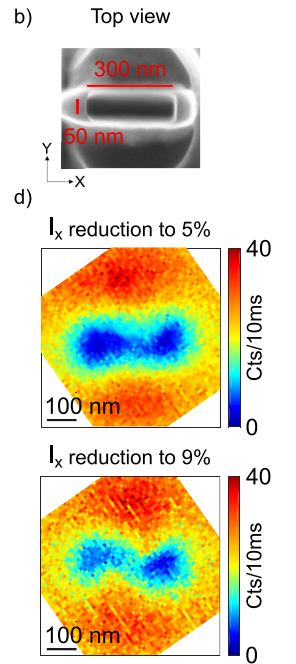

$\mathrm{I}_{\mathrm{x}}$ reduction to $21 \%$

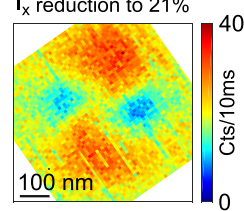

I reduction to $77 \%$
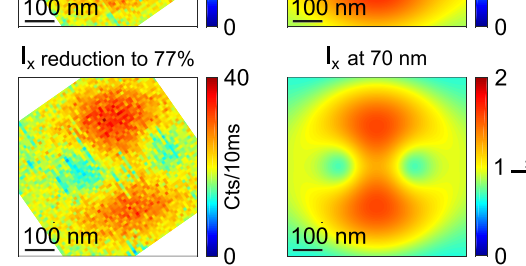

$\mathrm{I}_{\mathrm{x}}$ at $70 \mathrm{~nm}$

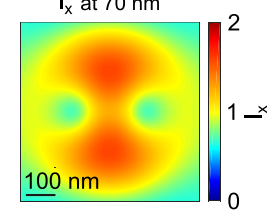

f)

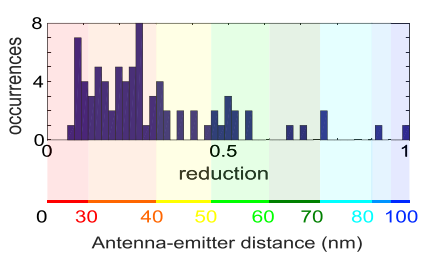

Figure 4. Single DBT molecule near-field hot and cold-spots mapping. (a, b) SEM images of the resonant dipole antenna (200 $\mathrm{nm}$ long) and the detuned nanoantenna (300 nm long) fabricated by ion-beam milling. (c) Measured fluorescence (left) and simulated (right) intensity maps of dipole antenna scans over a single DBT molecule, for different antenna-DBT distances. The small offset due to antenna luminescence was subtracted. The measured maps show two bright spots of $50 \mathrm{~nm}$ fwhm at a distance of $220 \mathrm{~nm}$. (d) Measured (left) and simulated (right) intensity maps of detuned antenna scans over a single DBT molecule, for different antenna-DBT distances. The measured maps show two dark spots of 100 $\mathrm{nm}$ fwhm at a distance of $320 \mathrm{~nm}$. (e, f) Distributions of the measured near-field enhancement and reduction, for dipole and detuned nanoantenna, together with the corresponding antenna-emitter $z$ distances. The illumination wavelength is again $750 \mathrm{~nm}$. The $x$-field intensities are normalized to the incoming intensity. The DBT molecules are oriented along the $x$ direction, parallel to the nanoantenna rods.

providing $x$-oriented molecules, parallel to the antenna. The antenna is scanned in close proximity to the AC-flake at about $10 \mathrm{~nm} .{ }^{16}$ The DBT-antenna system is excited in a confocal arrangement, using a $1.3 \mathrm{NA}$ objective, with linearly $x$-polarized $750 \mathrm{~nm}$ light parallel to the antenna rod. The DBT fluorescence is collected by the same objective and detected by photon counting detectors (APD).

In Figure $4 c, d$ we show the measured field around the dipole and detuned antennas as probed by a DBT molecule for both antenna types, together with the FDTD simulated near-field maps. For the dipolar antenna, we observe two bright spots of $50 \mathrm{~nm}$ fwhm, separated by $220 \mathrm{~nm}$, in agreement with the FDTD simulations. Comparing the measured excitation enhancement to the calculated values, we estimate the relative antenna-emitter distance $(z)$ : the enhancements of $2.5 \times, 2.1 \times$, $1.5 \times$, and $1.1 \times$ (top to bottom) correspond to the $z=20,22$, 25 , and $30 \mathrm{~nm}$ distance of the DBT molecule. As expected, with increasing depth $z$, the antenna effect becomes progressively weaker. Notably, for the detuned antenna we observe the opposite behavior. Figure $4 \mathrm{~d}$ reveals the manifestation of two dark spots, $100 \mathrm{~nm}$ fwhm, separated by $320 \mathrm{~nm}$, the first direct experimental demonstration of the predicted cold spots. Also, we notice the presence of two extended bright lobes at $\lambda / 2$ distance from the center. Both the dark spots and halo are due to near-field interference, as confirmed by the simulations on the right. In the cold-spot area, the DBT fluorescence is strongly reduced, corresponding to an excitation intensity of only $5 \%$. From top to bottom, we present antenna scans over different DBT molecules, with excitation reductions to respectively $5 \%, 9 \%, 21 \%$, and $77 \%$, corresponding to $z \sim 15,30,40$, and $70 \mathrm{~nm}$. The destructive near-field interference is most effective at short distance and fades out at the longer distance $z$. Interestingly, the further away constructive interference lobes at $\lambda / 2$ distance grow 

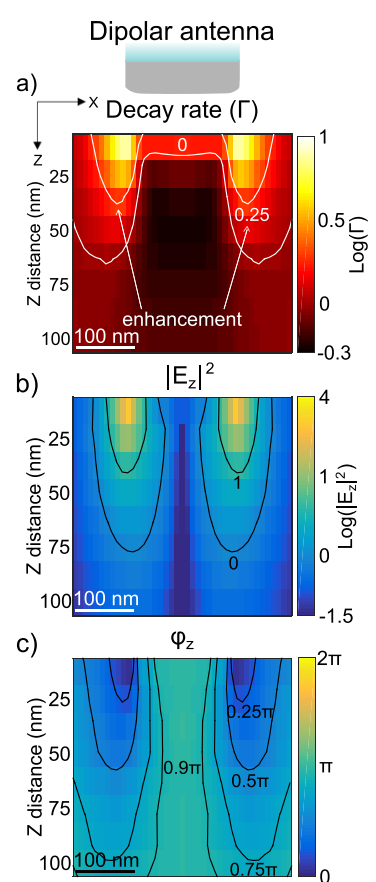

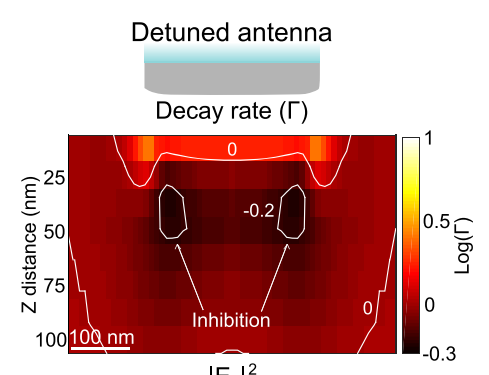

$\left|E_{z}\right|^{2}$

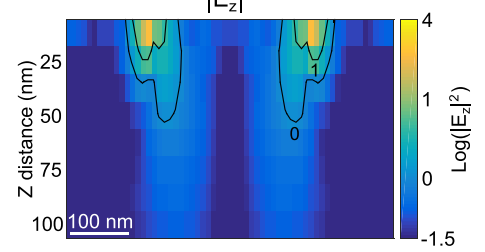

$\varphi_{\mathrm{z}}$

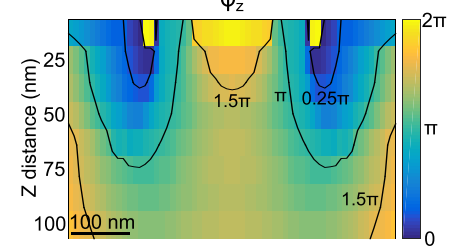

d)
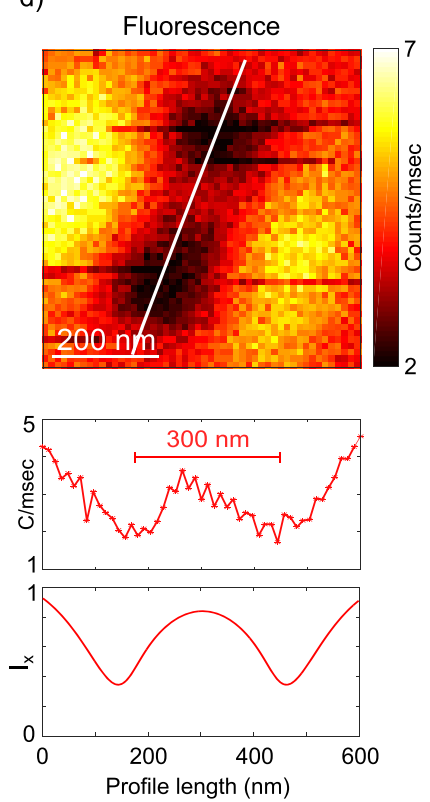

e)

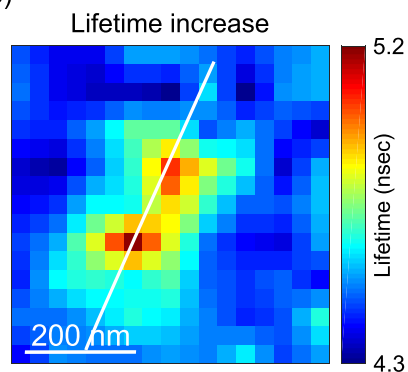

Figure 5. Spontaneous emission inhibition of a single molecule by a detuned antenna. (a) FDTD simulations of the decay rate $\Gamma$ of an $x$-oriented emitter positioned under a dipole and detuned antenna. The $\Gamma$ (in log-scale) is relative to the $\Gamma$ for the free emitter. For the dipole antenna $\Gamma$ is enhanced in two lobes located in proximity to the antenna ends. For the detuned antenna zones of inhibition, decreased $\Gamma \phi$ appear at a typical distance of 40-50 nm from the antenna, in two minima separated by around $200 \mathrm{~nm}$. (b) Calculated scattered $z$-field intensity (log-scale) with the unity intensity contour indicated. (c) Calculated $z$-field phase, showing the $\pi$-phase contour close to the detuned antenna. (d) Experimental fluorescence map of a single DBT molecule showing two dark spots, $100 \mathrm{~nm}$ fwhm, separated $300 \mathrm{~nm}$. The fluorescence response matches well the calculation (red profiles). (e) Corresponding fluorescence lifetime image exhibiting two maxima of $16 \%$ lifetime increase, $100 \mathrm{~nm}$ fwhm, separated $200 \mathrm{~nm}$. The measured lifetime profile along the antenna axis (white line) matches well the calculation for $z=50 \mathrm{~nm}$ (blue profiles). Simulations performed for an emitter at $780 \mathrm{~nm}$ wavelength, with an internal quantum efficiency of 1 .

toward the center area for increasing $z$-distance, until bridging the center at $70 \mathrm{~nm}$ distance, in perfect agreement between experiment and theory. Panels e and $\mathrm{f}$ of Figure 4 show the distribution of measured excitation enhancement/suppression for different molecules. The maximum excitation enhancement for the dipole antenna is $8 \times$, while the average value is $2.4 \times$, corresponding to calculated $z$-distances of $\sim 8$ and $15 \mathrm{~nm}$, respectively. We only find excitation enhancement up to 25 $\mathrm{nm}$, consistent with simulations (Figure 2c). The maximum excitation reduction, for the detuned antenna, is down to $5 \%$ for a calculated $z$-distance of $\sim 15 \mathrm{~nm}$, while the mean reduced value is $28 \%$, for $z=40 \mathrm{~nm}$. In agreement with our simulations (Figure $2 \mathrm{c}$ ), the $z$-range affected by reduction extends much deeper toward $100 \mathrm{~nm}$, confirming that cold-spot generation is mediated by interference effects.

Now we consider control of spontaneous emission, exploiting the local phase tuning of nanoantennas. We have shown above that the local excitation field can be largely suppressed, using the proper choice of antenna detuning; therefore, it should equally be possible to create points in which a quantum emitter would experience a lower LDOS, i.e., inhibition of the spontaneous emission. Here we address the engineering of subwavelength areas in the space with increased or decreased radiative decay rate $\Gamma$, i.e., local spots with fast and slow decay.

In Figure $5 \mathrm{a}-\mathrm{c}$ we present FDTD simulations of the emission field generated by an $x$-oriented dipolar emitter, located under a $200 \mathrm{~nm}$ dipolar and $300 \mathrm{~nm}$ detuned antenna. Figure 5a shows the total decay rate change as a function of the antenna-emitter relative position in the $(x, z)$-plane. For both antennas, the $x$-oriented emitter experiences a strong decay rate increase in close proximity to the antenna ends. The fastdecay spots, of $50 \mathrm{~nm}$ fwhm, are separated 220 and $320 \mathrm{~nm}$ for both antennas, respectively. The fast-rate zones extend for several tenths of nanometers in depth, $\sim 60$ and $\sim 25 \mathrm{~nm}$, respectively, with a maximal rate increase (Purcell factor) of $8 \times$ and $2.6 \times$. For the detuned antenna case, we notice the presence of two distinct slow-decay spots, $100 \mathrm{~nm}$ in fwhm, separated by $200 \mathrm{~nm}$, at a depth of $\sim 40 \mathrm{~nm}$. In the spots, the relative decay rate is decreased to $\sim 0.55$ : local areas of spontaneous emission inhibition.

To appreciate the decay rate dependence on antenna resonance, panels $\mathrm{b}$ and $\mathrm{c}$ of Figure 5 show vertical $x-z$ maps of the scattered $z$-field intensity $I_{z, \text { scat }}$ (log-scale) and phase $\phi_{z}$ for the $x$-oriented dipole. $I_{z, \text { scat }}$ is particularly strong under the antenna ends, where $\phi_{z}$ is minimal, corresponding to the spots of enhanced rate. Notably, contour lines with $\phi_{z} \sim \pi$ occur as two distinct contours under the ends of the detuned antenna (Figure 5c). The spots of inhibition in Figure 5a correspond to the zones where the contour $\phi_{z} \sim \pi$ coincides with the scattering intensity close to unity in Figure $5 \mathrm{~b}$.

Finally, we return to the experiment and examine the changes in decay rate in the data presented in Figure 4. Panels $\mathrm{d}$ and e of Figure 5 present fluorescence and lifetime maps of a DBT molecule in close proximity to the $300 \mathrm{~nm}$ length detuned antenna (see the Supporting Information for lifetime analysis). The fluorescence map shows the cold spots, with the measured intensity profile nicely matching the calculation. The fluorescence is reduced to $40 \%$ corresponding to a DBT molecule at a depth of $z=50 \mathrm{~nm}$. Figure 5e shows the 
corresponding fluorescence lifetime map. Remarkably, two spots with increased lifetime show up, i.e., slow decay. At the outer zones of the lifetime map, where the DBT, we find a lifetime of $4.5 \mathrm{~ns}$ for the uncoupled case. In the central part with maximum antenna coupling the lifetime gets as long as 5.2 $\mathrm{ns}$, corresponding to an increase of $\sim 16 \%$. Looking at the lifetime profile along the antenna axis, one appreciates the two slow-decay spots with size $100 \mathrm{~nm}$ fwhm, separated by $200 \mathrm{~nm}$. The lifetime profile calculated for an $x$-oriented emitter located at $z=50 \mathrm{~nm}$ shows good agreement, with two spots at a distance of $200 \mathrm{~nm}$ from each other. The calculated lifetime increase of $1.5 \times$ is larger than measured. Theory assumes a perfect single crystalline antenna and a perfect molecule with $100 \%$ quantum efficiency ${ }^{30}$ The nanofabricated polycrystallinity of the antenna and slight no-radiative decay can easily reduce the lifetime enhancement.

In conclusion, we have presented the first complete study on the use of a local phase to control the nanoantenna coupling to a single photon emitter, in both excitation and emission. Varying the length of a nanorod antenna, we tuned away from resonance, to control the local phase. Exploiting near-field interference, we designed local zones with suppressed fields and decreased LDOS.

On the excitation side, we have shown for the first time a direct, optical visualization of subwavelength cold spots, with excitation intensity reduced to percent level, mapped with high resolution $(50 \mathrm{~nm})$ using a single molecule. Tuning the antenna off and on resonance switches the interference from cold- to hot-spot generation. Together with hot spots, the cold spots provide strong field contrast with high resolution and allow us to enhance the dynamic range to 3 orders of magnitude. They constitute alternative building blocks for full light manipulation at the nanoscale.

On the emission side, we have presented the first inhibition of spontaneous emission of a single molecule mediated by a metallic nanoantenna: slow-decay spots, with a lifetime increase by a factor $16 \%$. Compared to the inhibition close to a metallic surface (Drexhage ${ }^{3}$ ) or in a photonic crystal defect cavity, ${ }^{7-10}$ here we achieve inhibition in a subwavelength volume. It is remarkable that inhibition occurs in such an open and lossy nanoantenna-cavity system. ${ }^{31}$

The cold spots are an attractive alternative to high-resolution microscopy. Particularly, for localization microscopy of rapidly bleaching labels, the cold spots can act as a near-field alternative to the far-field MINFLUX super-resolution microscopy, ${ }^{32}$ with the advantage of superior resolution due to the subwavelength spatial response. Beyond high-resolution imaging, since cold spots provide an almost completely dark area located inside a diffraction limited confocal spot, they might find application in a study of energy transfer at the nanoscale. Additionally, the capability to influence the decay rate of a single photon emitter might be used to actively manipulate energy transfer at the nanoscale, with the potential to change energy paths in complex multichromophoric systems. Finally, the possibility to switch between the hotand cold-spot tuning antenna phase provides a direct knob for electric field gradient control at the subwavelength scale, with possible applications in the field of optical trapping and particle manipulation.

\section{ASSOCIATED CONTENT}

\section{Supporting Information}

The Supporting Information is available free of charge at https://pubs.acs.org/doi/10.1021/acs.nanolett.0c02607.

Preparation of DBT single molecule near-field samples; laser reflection and fluorescence images; antenna preparation; dependence of nanoantenna resonances on length; scanning antenna microscope; lifetime analysis; photon time-gated imaging and lifetime maps; $\mathrm{x}$-component scattered field phase horizontal maps; numerical simulations; the role of the $\mathrm{z}$-field component in the inhibition of emission; profiles of the scattered $\mathrm{z}$ field intensity and phase $\phi_{\mathrm{z}}$ (PDF)

\section{AUTHOR INFORMATION}

\section{Corresponding Author}

Niek F. van Hulst - ICFO, Institut de Ciences Fotoniques, The Barcelona Institute of Science and Technology, Castelldefels, Barcelona 08860, Spain; ICREA, Institució Catalana de Recerca i Estudis Avançats, Barcelona 08010, Spain; ○ orcid.org/0000-0003-4630-1776; Email: Niek.vanHulst@ ICFO.eu

\section{Authors}

Nicola Palombo Blascetta - ICFO, Institut de Ciences Fotoniques, The Barcelona Institute of Science and Technology, Castelldefels, Barcelona 08860, Spain; 이이.org/00000003-3827-5360

Pietro Lombardi - CNR-INO and LENS, European Laboratory for Non-Linear Spectroscopy, 50019 Firenze, Italy

Costanza Toninelli - CNR-INO and LENS, European Laboratory for Non-Linear Spectroscopy, 50019 Firenze, Italy; 다이.org/0000-0002-6843-058X

Complete contact information is available at: https://pubs.acs.org/10.1021/acs.nanolett.0c02607

\section{Author Contributions}

The experiments and data analysis were performed by N.P.B. N.P.B. and N.F.v.H. conceived the experiment. P.L. and C.T. provided the DBT in anthracene samples. All authors contributed to the manuscript. All authors have given approval to the final version of the manuscript.

\section{Funding}

N.F.v.H. acknowledges the financial support by the European Commission (ERC Advanced Grant 670949-LightNet), the Spanish Ministry of Science, Innovation \& Universities (MCIU/AEI: PGC2018-096875-B-I00), the Ministry of Economy ("Severo Ochoa" program for Centers of Excellence in R\&D SEV-2015-0522 and Plan Nacional FIS2015-69258P), the Catalan AGAUR (2017SGR1369), Fundació Privada Cellex, Fundació Privada Mir-Puig, and Generalitat de Catalunya through the CERCA program. N.P.B. acknowledges the Ministry of Economy (MINECO) for support of the FPI fellowship. C.T. thanks the COST Action Nanoscale Quantum Optics (MP1403) and acknowledges financial support from the MIUR program Q-Sec and Ente Cassa di Risparmio di Firenze (GRANCASSA). P.L. and C.T. acknowledge support from ERANET COFUND action PhotonicSensing (FASPEC project, PS-2016_22).

\section{Notes}

The authors declare no competing financial interest. 


\section{REFERENCES}

(1) Purcell, E. M. Spontaneous emission probabilities at radio frequencies. Phys. Rev. 1946, 69, 681.

(2) Drexhage, K. H.; Fleck, M.; Kuhn, H.; Schäfer, F. P.; Sperling, W. Beeinflussung der Fluoreszenz eins Europiumchelates durch einen Spiegel. Ber. Bunsenges. Phys. Chem. 1966, 70, 1179.

(3) Drexhage, K. H. Influence of a dielectric interface on fluorescence decay time. J. Lumin. 1970, 1-2, 693-701.

(4) Buchler, B. C.; Kalkbrenner, T.; Hettich, C.; Sandoghdar, V. Measuring the quantum efficiency of the optical emission of single radiating dipoles using a scanning mirror. Phys. Rev. Lett. 2005, 95, 063003.

(5) Chizhik, A. I.; Chizhik, A. M.; Khoptyar, D.; Bear, S.; Meixner, A. J.; Enderlein, J. Probing the radiative transition of single molecules with a tunable microresonator. Nano Lett. 2011, 11, 1700-1703.

(6) Frimmer, M.; Mohtashami, A.; Koenderink, A. F. Nanomechanical method to gauge emission quantum yield applied to nitrogen-vacancy centers in nanodiamond. Appl. Phys. Lett. 2013, 102, 121105.

(7) Yablonovitch, E. Inhibited Spontaneous Emission in Solid-State Physics and Electronics. Phys. Rev. Lett. 1987, 58, 2059-2062.

(8) John, S. Strong localization of photons in certain disordered dielectric superlattices. Phys. Rev. Lett. 1987, 58, 2486-2489.

(9) Lodahl, P.; van Driel, A. F.; Nikolaev, I. S.; Irman, A.; Overgaag, K.; Vanmaekelbergh, D.; Vos, W. L. Controlling the dynamics of spontaneous emission from quantum dots by photonic crystals. Nature 2004, 430, 654-657.

(10) Fujita, M.; Takahashi, S.; Tanaka, Y.; Asano, T.; Noda, S. Simultaneous Inhibition and Redistribution of Spontaneous Emission from Perovskite Photonic Crystals. Science 2005, 308, 1296-1299.

(11) Jakubczyk, T.; Franke, H.; Smolenski, T.; Sciesie, M.; Pacuski, W.; Golnik, A.; Schmidt-Grund, R.; Grundmann, M.; Kruse, C.; Hommel, D.; Kossacki, P. Inhibition and enhancement of the spontaneous emission of quantum dots in micropillar cavities with radial-distributed Bragg reflectors. ACS Nano 2014, 8, 9970-9978.

(12) Frimmer, M.; Koenderink, A. F. Spontaneous emission control in a tunable hybrid photonic system. Phys. Rev. Lett. 2013, 110, 217405 .

(13) Özelci, E.; et al. Quantitative Measurements of the pHSensitive Quantum Yield of Fluorophores in Mesoporous Silica Thin Films Using a Drexhage-Type Experiment. J. Phys. Chem. C 2019, 123, 20468-20475.

(14) Kuhn, S.; Hakanson, U.; Rogobete, L.; Sandoghdar, V. Enhancement of single-molecule fluorescence using a gold nanoparticle as an optical nanoantenna. Phys. Rev. Lett. 2006, 97, 017402.

(15) Hoang, T. B.; Akselrod, G. M.; Mikkelsen, M. H. Ultrafast Room-Temperature Single Photon Emission from Quantum Dots Coupled to Plasmonic Nanocavities. Nano Lett. 2016, 16, 270-275.

(16) Singh, A.; de Roque, P. M.; Calbris, G.; Hugall, J. T.; van Hulst, N. F. Nanoscale Mapping and Control of Antenna-Coupling Strength for Bright Single Photon Sources. Nano Lett. 2018, 18, 2538-2544.

(17) Bouchet, D.; Mivelle, M.; Proust, J.; Gallas, B.; Ozerov, I.; Garcia-Parajo, M. F.; Gulinatti, A.; Rech, I.; De, Wilde Y.; Bonod, N.; Krachmalnicoff, V.; Bidault, S. Enhancement and Inhibition of Spontaneous Photon Emission by Resonant Silicon Nanoantennas. Phys. Rev. Appl. 2016, 6, 064016.

(18) Hoogenboom, J. P.; Sanchez-Mosteiro, G.; Colas, des Francs G.; Heinis, D.; Legay, G.; Dereux, A.; van Hulst, N. F. The single molecule probe: Nanoscale vectorial mapping of photonic mode density in a metal nanocavity. Nano Lett. 2009, 9, 1189-1195.

(19) Chikkaraddy, R.; de Nijs, B.; Benz, F.; Barrow, S. J.; Scherman, O. A.; Rosta, E.; Demetriadou, A.; Fox, P.; Hess, O.; Baumberg, J. J. Single-molecule strong coupling at room temperature in plasmonic nanocavities. Nature 2016, 535, 127-130.

(20) Hugall, J. T.; Singh, A.; van Hulst, N. F. Plasmonic Cavity Coupling. ACS Photonics 2018, 5, 43-53.

(21) Koenderink, A. F. Single-Photon Nanoantennas. ACS Photonics 2017, 4, 710-722.
(22) Istrate, E.; Sargent, E. H. Measurement of the phase shift upon reflection from photonic crystals. Appl. Phys. Lett. 2005, 86, 151112.

(23) Arcari, M.; Sollner, I. J. A.; Lindskov, Hansen S.; Mahmoodian, S.; Liu, J.; Thyrrestrup, H. L. E. H.; Song, J. D.; Stobbe, S.; Lodahl, P. Near-Unity Coupling Efficiency of a Quantum Emitter to a Photonic Crystal Waveguide. Phys. Rev. Lett. 2014, 113, 093603.

(24) Lecamp, G.; Lalanne, P.; Hugonin, J. P. Very large spontaneous-emission $\beta$ factors in photonic-crystal waveguides. Phys. Rev. Lett. 2007, 99, 023902.

(25) Curto, A. G.; Volpe, G.; Taminiau, T. H.; Kreuzer, M. P.; Quidant, R.; van Hulst, N. F. Unidirectional emission of a quantum Dot Coupled to a Nanoantenna. Science 2010, 329, 930-933.

(26) Yu, N.; Genevet, P.; Kats, M. A.; Aieta, F.; Tetienne, J.; Capasso, F.; Gaburro, Z. Light propagation with phase discontinuities: generalized laws of reflection and refraction. Science 2011, 334, 333337.

(27) Haggui, M.; Dridi, M.; Plain, J.; Marguet, S.; Perez, H.; Schatz, G. C.; Wiederrecht, G. P.; Gray, S. K.; Bachelot, R. Spatial confinement of electromagnetic hot and cold spots in gold nanocubes. ACS Nano 2012, 6, 1299-1307.

(28) Toninelli, C.; Early, K.; Bremi, J.; Renn, A.; Gotzinger, S.; Sandoghdar, V. Near-infrared single-photons from aligned molecules in ultrathin crystalline films at room temperature. Opt. Express 2010, $18,6577-6582$.

(29) Nicolet, A. A. L.; Bordat, P.; Hofmann, C.; Kolchenko, M. A.; Kozankiewicz B, Ross B.; Orrit, M. Single dibenzoterrylene molecules in an anthracene crystal: Main insertion sites. ChemPhysChem 2007, 8, $1929-1936$

(30) Major, K. D.; Lien, Y.; Polisseni, C.; Grandi, S.; Wei, Kho K.; Clark, A. S.; Hwang, J.; Hinds, E. A. Growth of optical-quality anthracene crystals doped with dibenzoterrylene for controlled single photon production. Rev. Sci. Instrum. 2015, 86, 083106.

(31) Hadad, Y.; Engheta, N. Possibility for inhibited spontaneous emission in electromagnetically open parity-time-symmetric guiding structures. Proc. Natl. Acad. Sci. U. S. A. 2020, 117 (11), 5576-5581.

(32) Balzarotti, F.; Eilers, Y.; Gwosch, K. C.; Gynna, A. H.; Westphal, V.; Stefani, F. D.; Elf, J.; Hell, S. W. Nanometer resolution imaging and tracking of fluorescent molecules with minimal photon fluxes. Science 2017, 355, 606-612. 